\title{
Two novel heterozygous variants in ATP1A3 cause movement disorders
}

\author{
Shogo Furukawa (D) $^{1}$, Sachiko Miyamoto ${ }^{1}$, Shinobu Fukumura ${ }^{2}$, Kazuo Kubota ${ }^{3,4}$, Toshiaki Taga ${ }^{5}$, Mitsuko Nakashima ${ }^{1}{ }^{1}$ and \\ Hirotomo Saitsu iD ${ }^{1 \text { 网 }}$
}

(c) The Author(s) 2022

\begin{abstract}
Variants in ATP1A3 cause neuropsychiatric disorders, especially those characterized by movement disorders. In this study, we performed whole exome sequencing for two patients with movement disorders and identified two novel heterozygous ATP1A3 variants, a missense c.2408G >A variant and an indel c.2672_2688+10delinsCAG variant. The unique indel variant occurred at the exon-intron boundary at the $3^{\prime}$ end of exon 19 , and mRNA analysis revealed that this variant caused in-frame indel alteration at the Ser891_Trp896 residue.
\end{abstract}

Human Genome Variation (2022) 9:1-4; https://doi.org/10.1038/s41439-022-00184-y

The $\mathrm{Na}^{+} / \mathrm{K}^{+}$-ATPase transmembrane ion pump is mainly composed of two subunits, a large catalytic subunit (alpha) and a smaller glycoprotein subunit (beta). In humans, $\mathrm{Na}^{+} / \mathrm{K}^{+}$-ATPase has four different a-subunit isoforms, especially the $a_{2}$-isoform and $a_{3}$-isoform (encoded ATP1A2 and ATP1A3), which are mainly expressed in the brain ${ }^{1}$. To date, only these two isoforms have been shown to be related to neurological diseases in humans ${ }^{2,3}$. Heterozygous variants in ATP1A3 cause rapid-onset dystonia parkinsonism (RDP, OMIM \# 128235) ${ }^{2}$, alternating hemiplegia of childhood (AHC, OMIM \# 614820) ${ }^{4}$, cerebellar ataxia, areflexia, pes

Table 1. Summary of the clinical and genetic findings in the patients.

\begin{tabular}{|c|c|c|}
\hline & Patient 1 & Patient 2 \\
\hline Age & $1 \mathrm{y} 0 \mathrm{~m}$ & $14 y$ \\
\hline Onset & $0 y 4 m$ & $1 \mathrm{y} 9 \mathrm{~m}$ \\
\hline Sex & Female & Male \\
\hline Dystonia & - & + \\
\hline Hemiplegia/Quadriplegia & - & - \\
\hline Epilepsy & - & - \\
\hline Abnormal eye movement & - & + \\
\hline Developmental delay & + & - \\
\hline \multicolumn{3}{|l|}{ ATP1A3 variants } \\
\hline CDNA change & c. $2408 \mathrm{G}>\mathrm{A}$ & c. $2672 \_2688+10$ delinsCAG \\
\hline Amino acid change & p.(Gly803Asp) & p.Ser891_Trp896delinsThrAlaGlyCysCysValSerAlaHisArgLyslleProGly \\
\hline SIFT & 0 & N/A \\
\hline PolyPhen-2 & 0.973 & N/A \\
\hline CADD & 27.6 & N/A \\
\hline M-CAP & 0.962096 & $\mathrm{~N} / \mathrm{A}$ \\
\hline \multirow[t]{2}{*}{ ACMG guideline } & Pathogenic & Likely pathogenic \\
\hline & PS2, PM1, PM2, PP3, PP5 & PS3, PM2, PM4 \\
\hline
\end{tabular}

Variat description based on NM_152296.5.

PolyPhen-2 Polymorphism Phenotyping v2, CADD Combined Annotation Dependent Depletion, SIFT Sorting Intolerant From Tolerant, M-CAP Mendelian Clinically Applicable Pathogenicity, ACMG American College of Medical Genetics and Genomics.

\footnotetext{
${ }^{1}$ Department of Biochemistry, Hamamatsu University School of Medicine, Hamamatsu, Japan. ${ }^{2}$ Department of Pediatrics, Sapporo Medical University School of Medicine, Sapporo, Japan. ${ }^{3}$ Department of Pediatrics, Gifu University Graduate School of Medicine, Gifu, Japan. ${ }^{4}$ Division of Clinical Genetics, Gifu University Hospital, Gifu, Japan. ${ }^{5}$ Department of Pediatrics, Nagahama City Hospital, Shiga, Japan. ${ }^{凶}$ email: hsaitsu@hama-med.ac.jp
}

Received: 15 November 2021 Revised: 5 January 2022 Accepted: 5 January 2022

Published online: 18 February 2022 


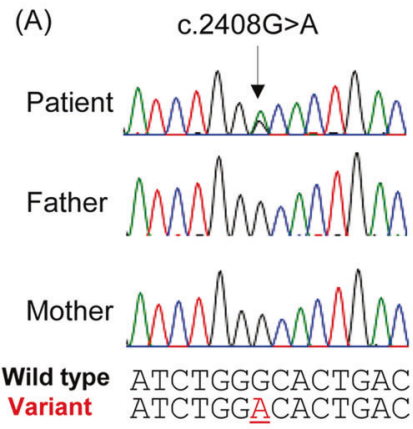

(C)

Wild type (WT)

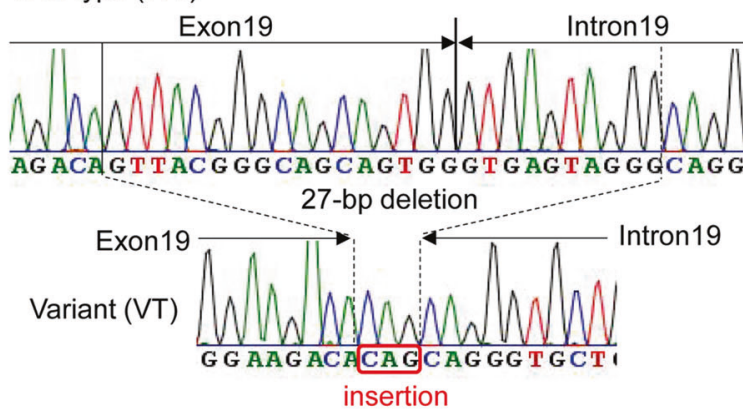

(E)$$
\text { insertion }
$$

c.2672_2688+10delinsCAG

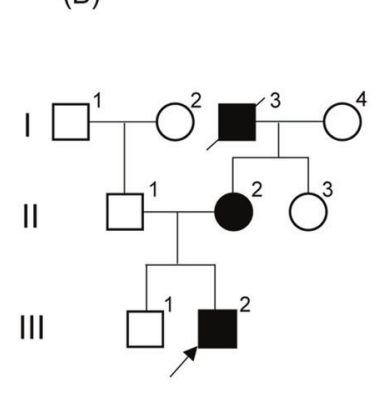

(D)

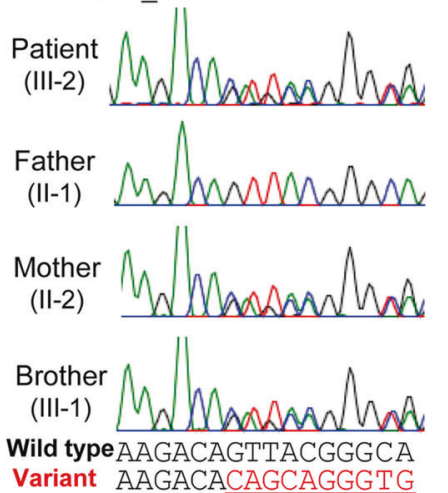

WT

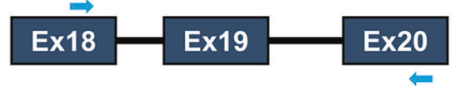

VT
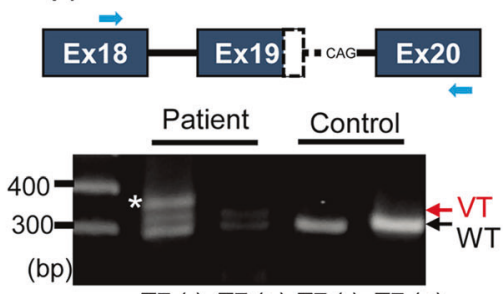

T7 (-) T7 (+) T7 (-) T7 (+)

* heteroduplex

Exon20

17-bp deletion
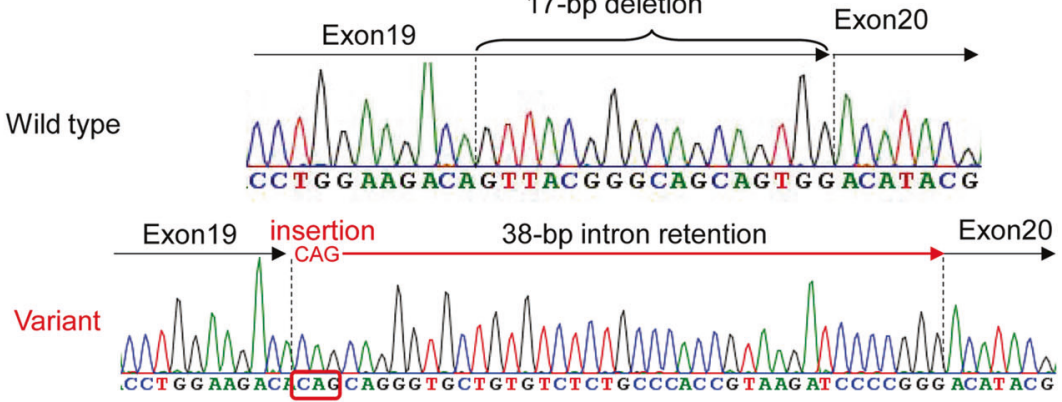

\footnotetext{
890891892893894895896897898899900901902903904905906907908909

WT

Asp Ser Tyr Gly Gln Gln Trp Thr Tyr Glu Gln Arg Lys Val Val Glu Phe Thr Cys $\overline{\text { His }}$

GAC AGT TAC GGG CAG CAG TGG ACA TAC GAG CAG AGG AAG GTG GTG GAG TTC ACC TGC CAC

GAC ACA GCA GGG TGC TGT GTC TCT GCC CAC CGT AAG ATC CCC GGG ACA TAC GAG CAG AGG

VT Asp Thr Ala Gly Cys Cys Val Ser Ala His Arg Lys lle Pro Gly Thr Tyr Glu Gln Arg
}

Fig. 1 Electropherograms of two family samples. A Sanger sequencing reveals that the c.2408G>A variant (arrow) occurred de novo. B Family pedigree and electropherogram results of family samples. Sanger sequencing denotes that the c.2672_2688+10delinsCAG variant is inherited from patient 2's affected mother. C Electropherograms of wild-type (top) and variant (bottom) PCR clones of the proband. A 27-bp deletion (dashed lines) and 3-bp (CAG) insertion (red square) occurred at the exon 19 - intron 19 boundary in the variant allele. D Schematic representation of the ATP1A3 gene structure and electrophoresis of RT-PCR analysis using cDNA derived from the patient and a control. Black boxes, lines and light blue arrows denote the coding exons, introns and RT-PCR primers, respectively. The patient showed two bands of different sizes (black and red arrows). The largest sized band (white asterisk) found in the patient was digested with T7 endonuclease I, indicating heteroduplexes. WT, wild-type, VT, variant. (E) CDNA sequences (upper) and amino acid sequences (lower) of WT and variant. Electropherograms of the variant show a 17-bp deletion (dashed line) at the $3^{\prime}$ end of exon 19 and a 3-bp (CAG) insertion and 38-bp intron retention of the $5^{\prime}$ end of intron 19 (red arrow). This indel variant caused in-frame amino acid alteration, and 6 amino acid residues of WT (from Ser891 to Trp896, blue box) were replaced with another 14 amino acids (red box).

cavus, optic atrophy, sensorineural hearing loss (CAPOS) syndrome (OMIM \# 601338), and polymicrogyria ${ }^{5,6}$. Here, we report two novel variants in ATP1A3 (NM_152296.5) found in patients with movement disorders.

The first patient (patient 1) is a 1-year-old Japanese girl born as a third child of nonconsanguineous healthy parents without asphyxia. Signs of developmental delay were recognized at 4 months with unstable head control. Neurological examination at 5 months revealed poor head control and hypotonia. At 7 months, she started to show foot clonus, hyperreflexia of the patellar tendon and the Achilles tendon, but there was an absence of alternating paralysis, seizures and involuntary movements. 
Ophthalmological examination at 5 months indicated loss of eye pursuit and normal light reflex but no abnormal eye movements. Biochemical analysis and electroencephalogram (EEG) examination were normal. Brain magnetic resonance imaging (MRI) at one year showed hypoplasia of the brainstem.

The second patient (patient 2) is a 14-year-old Japanese boy who was born as the second child without abnormal perinatal history. His mother and maternal grandfather also developed mild adult onset dystonia of the upper extremities at approximately 40 and 55 years of age, respectively. At 1 year and 9 months, he suddenly developed throwing head back, turning eyes upward, and weakness of the upper limbs lasting several tens of minutes. At 5 years, he began to show gradual, paroxysmal dystonic attacks in his left hand. His muscle stiffness was noticeable outside of the attacks and worsened when he was nervous since 12 years of age. Brain MRI, spinal fluid test, somatosensory evoked potentials, and short-term EEG were normal. Long-term EEG and electromyogram at 12 years showed sudden onset of left-hand dystonia with stress and caffeine load without EEG response.

This study was approved by the Institutional Review Board Committee at Hamamatsu University School of Medicine. After receiving written informed consent, we performed whole exome sequencing (WES) as described previously ${ }^{7,8}$. WES detected two novel ATP1A3 variants, a missense variant (NM_152296.5: c.2408G >A, p.(Gly803Asp)) and an indel variant (NM_152296.5: c.2672_2688+10delinsCAG), in patients 1 and 2 , respectively (Table 1). These variants were not observed in the Genome Aggregation Database v3.1.1 (accessed Aug 2021), 8.3KJPN Allele Frequency Panel (https://jmorp.megabank.tohoku.ac.jp/) or our inhouse 218 control exome data. Sanger sequencing revealed that the c.2408G >A variant occurred de novo (Fig. 1A), and multiple in silico prediction tools predicted it to be deleterious. Although the c.2408G >A variant has been registered in ClinVar as a pathogenic variant (RCV000525007), detailed clinical features have not been reported. The c.2672_2688+10delinsCAG variant was inherited from his affected mother and found in his elder brother without symptoms (Fig. 1B). The c.2672_2688+10delinsCAG variant consists of a 27-bp deletion spanning a part of exon 19 (17 bp) and intron 19 (10 bp) and a 3-bp (CAG) insertion (Fig. 1C), suggesting that it can cause abnormal splicing. We performed reverse transcription polymerase chain reaction (RT-PCR) using total RNA extracted from peripheral leukocytes derived from patient 2 and a healthy control as previously described ${ }^{7}$. Two different-sized products were amplified in the patient 2 sample (Fig. 1D). Sequencing analysis of cloned PCR products revealed 38bp intron retention of intron 19 along with 17-bp deletion and 3-bp insertion, leading to in-frame alteration, p. Ser891_Trp896delinsThrAlaGlyCysCysValSerAlaHisArgLyslleProGly: Six amino acid (from Ser891 to Trp896) residues were replaced by another 14 amino acids in the ATP1A3 protein (Fig. 1E). Based on the American College of Medical Genetics and Genomics standards and guidelines, the c.2408G $>$ A and c.2672_2688+10delinsCAG variants were classified as pathogenic and likely pathogenic, respectively (Table 1$)^{9}$.

Patients with deleterious ATP1A3 variants shared similar clinical phenotypes, but there were some differences in patients with AHC and RDP. When comparing RDP with $\mathrm{AHC}$, patients with $\mathrm{AHC}$ often have paroxysmal episodes and develop symptoms at a younger age (almost $<18$ months) ${ }^{2,3}$. Patient 1 showed early onset developmental delay and hypotonia, whereas she also showed atypical findings, including early-onset pyramidal signs and brainstem hypoplasia. Recent studies suggested that AHCrelated variants clustered at the transmembrane regions and functional domains, and variants located in exons $8,14,17$, and 18 tend to be associated with more severe phenotypes ${ }^{10,11}$. Patient 1 had the c.2408G>A, p.(Gly803Asp) variant located at exon 17 in the transmembrane region, consistent with previous studies.
Meanwhile, patient 2 had the c.2672_2688+10delinsCAG, p. Ser891_Trp896delinsThrAlaGlyCysCysValSerAlaHisArgLyslleProGly variant located at the extracellular region of ATP1A3. Recent studies have indicated that both missense and in-frame variants in this extracellular region cause various types of neurological disorders, including AHC, CAPOS, and polymicrogyria ${ }^{6,12}$. Patient 2 showed an intermediate phenotype between RDP and AHC due to the relatively late onset and the presence of episodic symptoms. His mother and maternal grandfather showed a typical and mild clinical course of RDP, including late onset onesided limb dystonia that worsened when they were nervous and parkinsonism that was observed in the grandfather. Meanwhile, his elder brother, two years older than patient 2 , has not yet developed any neurological symptoms. These findings suggested that this splicing variant might cause RDP with variable severity. The genotype-phenotype correlation and penetrance of ATP1A3related disorders are variable ${ }^{13,14}$, and some familial cases have a broad range of severities ${ }^{15,16}$. However, there are only a few reports showing phenotypic variability within families with ATP1A3 variants. Here, we precisely described the onset time and specific symptoms or severity for each individual in the family, clearly presenting an example of intrafamilial variability of ATP1A3related disorders.

\section{HGV DATABASE}

The relevant data from this Data Report are hosted at the Human Genome Variation Database at https://doi.org/10.6084/m9.figshare.hgv.3128, https://doi.org/10.6084/ m9.figshare.hgv.3131.

\section{REFERENCES}

1. McGrail, K. M. et al. Immunofluorescent localization of three Na,K-ATPase isozymes in the rat central nervous system: both neurons and glia can express more than one Na,K-ATPase. J. Neurosci. 11, 381-391 (1991).

2. de Carvalho Aguiar, $\mathrm{P}$. et al. Mutations in the $\mathrm{Na}^{+} / \mathrm{K}^{+}$-ATPase alpha3 gene ATP1A3 are associated with rapid-onset dystonia parkinsonism. Neuron 43, 169-175 (2004).

3. Swoboda, K. J. et al. Alternating hemiplegia of childhood or familial hemiplegic migraine? A novel ATP1A2 mutation. Ann. Neurol. 55, 884-887 (2004).

4. Heinzen, E. L. et al. De novo mutations in ATP1A3 cause alternating hemiplegia of childhood. Nat. Genet. 44, 1030-1034 (2012).

5. Demos, M. K. et al. A novel recurrent mutation in ATP1A3 causes CAPOS syndrome. Orphanet J. Rare Dis. 9, 15 (2014).

6. Miyatake, S. et al. De novo ATP1A3 variants cause polymicrogyria. Sci. Adv. 7, eabd2368 (2021).

7. Hiraide, T. et al. De novo variants in SETD1B cause intellectual disability, autism spectrum disorder, and epilepsy with myoclonic absences. Epilepsia Open. 4, 476-481 (2019).

8. Miyamoto, S. et al. A case of de novo splice site variant in SLC35A2 showing developmental delays, spastic paraplegia, and delayed myelination. Mol. Genet. Genom. Med. 7, e814 (2019).

9. Richards, S. et al. Standards and guidelines for the interpretation of sequence variants: a joint consensus recommendation of the American College of Medical Genetics and Genomics and the Association for Molecular Pathology. Genet Med. 17, 405-424 (2015).

10. Yang, X. et al. ATP1A3 mutations and genotype-phenotype correlation of alternating hemiplegia of childhood in Chinese patients. PLOS ONE 9, e97274 (2014).

11. Rosewich, $\mathrm{H}$. et al. The expanding clinical and genetic spectrum of ATP1A3related disorders. Neurology 82, 945-955 (2014).

12. Gurrieri, F. et al. Recognizable facial features in patients with alternating hemiplegia of childhood. Am. J. Med. Genet. A 170, 2698-2705 (2016).

13. Roubergue, A. et al. The multiple faces of the ATP1A3-related dystonic movement disorder. Mov. Disord. 28, 1457-1459 (2013).

14. Brashear, A. et al. ATP1A3 mutations in infants: a new rapid-onset dystoniaParkinsonism phenotype characterized by motor delay and ataxia. Dev. Med. Child Neurol. 54, 1065-1067 (2012).

15. Brashear, A. et al. The phenotypic spectrum of rapid-onset dystonia-parkinsonism (RDP) and mutations in the ATP1A3 gene. Brain 130, 828-835 (2007).

16. Potic, A. et al. CAPOS syndrome and hemiplegic migraine in a novel pedigree with the specific ATP1A3 mutation. J. Neurol. Sci. 358, 453-456 (2015). 


\section{ACKNOWLEDGEMENTS}

We would like to thank the patient's family for participating in this work. We also thank K. Shibazaki, M. Tsujimura, and A. Kitamoto for their technical assistance. This work was supported in part by the Japan Society for the Promotion of Science, KAKENHI (Grant number JP $20 \mathrm{H} 03641$ (H.S.) and JP21K06819 (M.N.), the Takeda Science Foundation (M.N. and H.S.), the Mochida Memorial Foundation for Medical and Pharmaceutical Research (M.N.), and HUSM Grant-in-Aid from Hamamatsu University School of Medicine (S.F., M.N., and H.S.).

\section{COMPETING INTERESTS}

The authors declare no competing interests.

\section{ADDITIONAL INFORMATION}

Correspondence and requests for materials should be addressed to Hirotomo Saitsu.

Reprints and permission information is available at http://www.nature.com/ reprints
Publisher's note Springer Nature remains neutral with regard to jurisdictional claims in published maps and institutional affiliations.

\section{(c) (i)}

Open Access This article is licensed under a Creative Commons Attribution 4.0 International License, which permits use, sharing, adaptation, distribution and reproduction in any medium or format, as long as you give appropriate credit to the original author(s) and the source, provide a link to the Creative Commons license, and indicate if changes were made. The images or other third party material in this article are included in the article's Creative Commons license, unless indicated otherwise in a credit line to the material. If material is not included in the article's Creative Commons license and your intended use is not permitted by statutory regulation or exceeds the permitted use, you will need to obtain permission directly from the copyright holder. To view a copy of this license, visit http://creativecommons. org/licenses/by/4.0/.

(c) The Author(s) 2022 